\title{
A ReView on Distributed Control of COOPERATING MINI UAVS
}

\author{
Qasim Ali, Nils Gageik and Sergio Montenegro \\ Chair Aerospace Information Technology, University of Wuerzburg, Germany
}

\begin{abstract}
Mini Unmanned Aerial Vehicles (MUAVs) are becoming popular research platform and drawing considerable attention, particularly during the last decade due to their multi-dimensional applications in almost every walk of life. MUAVs range from simple toys found at electronic supermarkets for entertainment purpose to highly sophisticated commercial platforms performing novel assignments like offshore wind power station inspection and 3D modelling of buildings. This paper presents an overview of the main aspects in the domain of distributed control of cooperating MUAVs to facilitate the potential users in this fascinating field. Furthermore it gives an overview on state of the art in MUAV technologies e.g. Photonic Mixer Devices (PMD) camera, distributed control methods and on-going work and challenges, which is the motivation for many researchers all over the world to work in this field.
\end{abstract}

\section{KEYWORDS}

Mini Unmanned Aerial Vehicle, Distributed Control, Cooperative UAV, Autonomous UAV, Mobile Sensor Network

\section{INTRODUCTION}

MUAVs like Quadrocopter (Figure 1) can be regarded as flying robots that may operate in threedimensional space. The biggest advantages of MUAVs include their much lower cost compared with manned vehicles, risk avoidance for human pilots and their remote sensing capabilities. Single MUAV may be used for forest fire monitoring and flood damage assessment etc. However, some other interesting applications are envisaged that may not be performed by a single MUAV and necessitate the use of multiple units. Such valuable applications include Cooperative Transportation, Aerial Mapping, Search and Rescue, Communication Relays and Air Refuelling etc.

These platforms are becoming more and more versatile as the sensors are miniaturized and onboard computing power is enhanced. Although these vehicles have several advantages compared with manned platforms, however control requirements are comparatively more stringent and therefore generally require more sophisticated control techniques. Cooperative control for MUAVs poses strict performance requirements like task coupling, robustness, optimality and scalability [1]. Highly non-linear flight dynamics of MUAVs make it demanding to determine real-time relative position and attitude vis-a-vis data latency and communication data packets loss.

DOI : 10.5121/ijaia.2014.5401 


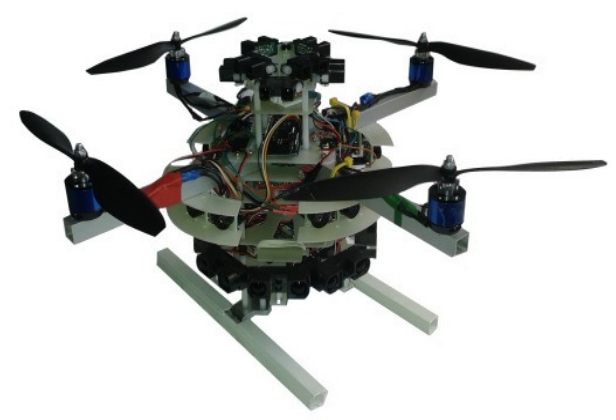

Figure 1. Quadrocopter

Cooperation behaviour of units implies some degree of coupling among the units. Generally speaking, greater the degree of coupling, more challenging it is to formulate effective cooperative solutions [2]. This becomes more obvious taking into consideration that a swarm of MUAVs need to cooperate in a way that it can rescue a person from a burning house as depicted in Figure 2.

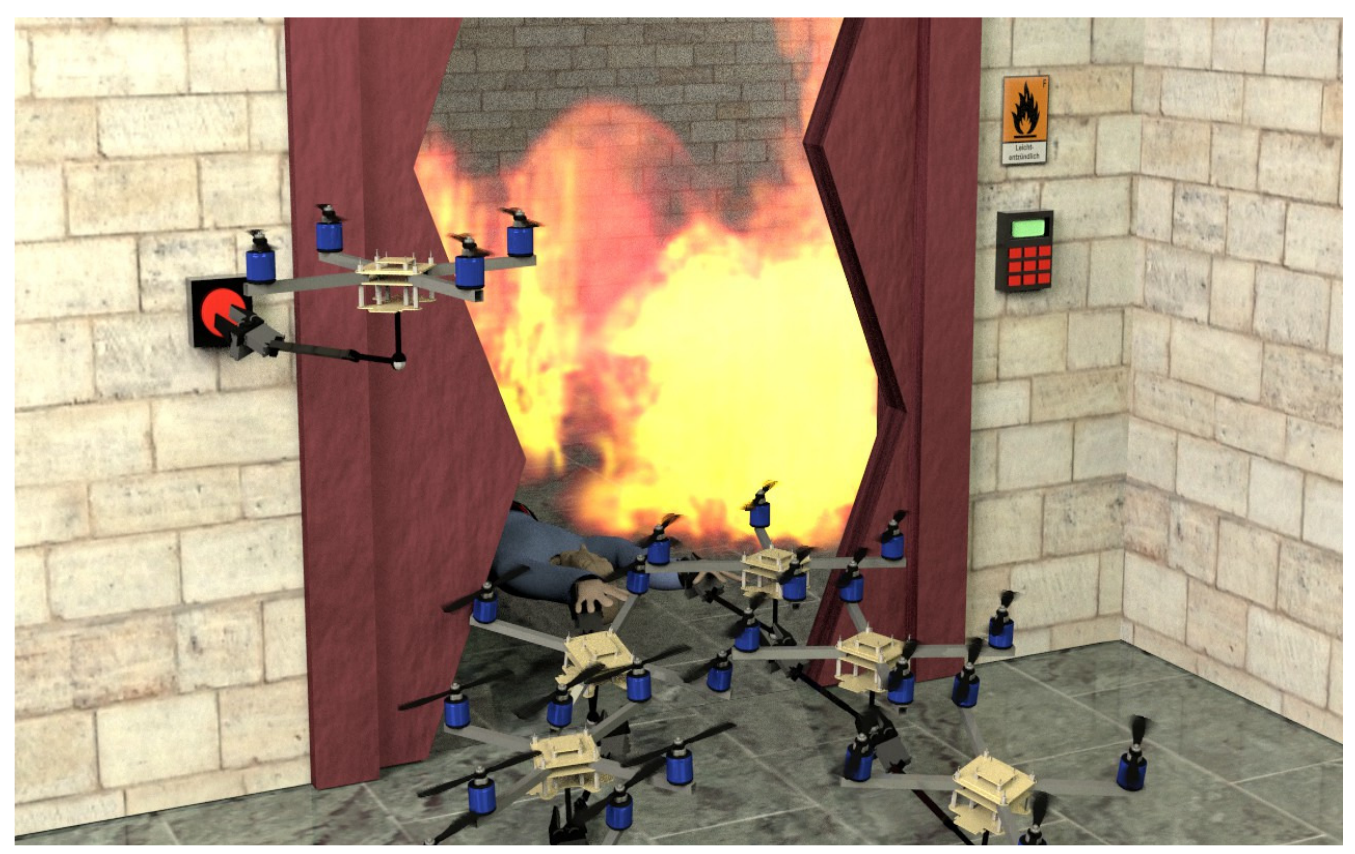

Figure 2. Cooperative emergency quadrocopters

Small UAVs are divided into two major categories; mini UAV and micro UAV. Mini UAV wing span is between $0.5-2$ meters, while micro UAV is between $0.1-0.5$ meters [3]. Our survey encompasses both mini and micro UAVs. These platforms may take different forms like fixed wing or Vertical Take Off and Landing (VTOL) devices including Twinrotor, Trirotor, Quadrotor, Hexacopter, Octocopter etc. Quadcopters are most popular due to their agility and ease of construction. Due to off-center location of propellers, fast rotational dynamics are possible that may be exploited. VTOL devices have certain benefits compared with fixed wing, like more agility and no requirement for a take-off/ landing strip. Their drawbacks include less speed and endurance. Merits of fixed wing and VTOL are combined in the form of Tail-Sitters and Quad Tilt-Rotor convertible MAV [4]. 


\subsection{Constellation Architecture}

Selection of suitable constellation architecture is important for the formation control problem. Centralized strategies are usually easier to design than decentralized strategies [2]. However decentralized algorithms reduce the communication between units and improve the robustness. Mainly three types of architecture have been formulated in the literature namely, leader-follower, virtual structure, and behavioural approach [5].

\subsubsection{Leader-Follower}

Most of the multi-agent formation control researches have been carried out with leader-follower scheme. This is a concept taken from nature and an example can be seen in the formation flight of a birds swarm (Figure 3). With this approach, some vehicles are designated as leaders while others are treated as followers. For small formations, there may be only one leader. The states of the leader constitute the coordination variable, since the actions of the other vehicles in the formation are completely specified once the leader states are known [6]. This architecture is easier to understand and implement. However, this approach lacks robustness with respect to leader's failure. Though Virtual Leader strategy is also proposed to improve its robustness.

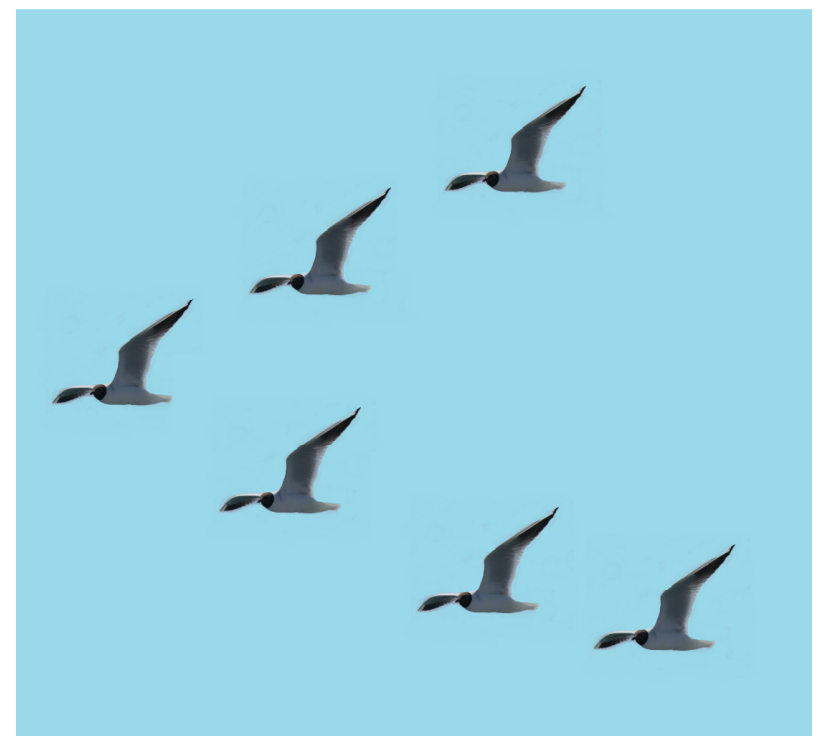

Figure 3. Birds in leader-follower formation

\subsubsection{Virtual Structure}

In this scheme, the entire formation is treated as a single virtual rigid body structure. Rather than following a path, each vehicle follows a moving point, which allows the virtual structure to potentially be attached to another vehicle. The guidance of a group is easier than other approaches since all agents in the formation are treated as a single object. But the formation can only perform synchronized manoeuvres, and it is difficult to consider obstacle avoidance.

\subsubsection{Behavioural Approach}

For Behaviour Approach, several desired behaviours are prescribed for each vehicle, including formation keeping, goal seeking, and collision/ obstacle avoidance. The control action of each 
International Journal of Artificial Intelligence \& Applications (IJAIA), Vol. 5, No. 4, July 2014

vehicle is a weighted average of the control for each behaviour. It is suitable for uncertain environments, but lack of a rigorous theoretic analysis. There are other approaches like [7] where a consensus based decentralized approach has been used for controller design. In order to provide redundancy, no explicit leader exists for such cases.

This paper is structured as follows; Section 2 describes the previous contributions made in this area. Section 3 informs about the sensors used/ to be used for MUAV cooperative flight. Section 4 covers the control techniques and relevant material. Section 5 describes the state of the art and the areas requiring attention in this domain.

\section{RELATED WORK}

The idea of formation flight of multi-units was inspired by the nature e.g. birds and bees. Though benefits of formation flight in terms of fuel saving have also been investigated [8]. A number of test-beds have been developed for testing and validation of formation flights [9] and control techniques for coordinated missions [10]. A lot has been done at Department of Aeronautics and Astronautics, Massachusetts Institute of Technology (MIT) in the domain of formation flight of micro UAVs in real time. For relative navigation, mainly Carrier Phase Differential GPS (CPDGPS) has been used exploiting pseudolite transmitters to provide accuracy of the order of few centimeters [11]. A mid-air rendezvous of two UAVs was also realized at MIT [12]. A methodology was proposed exploiting Dijkstra algorithm for real-time control of UAVs without having the knowledge of location of sites in a hostile territory [13]. A Simulink based controller was designed for autonomous aerial refuelling [14].

Load transportation with multiple quadcopters is useful when the load is heavier compared with the maximum thrust of a single quadcopter, or when additional redundancy is required for safety. But this is challenging since dynamically coupled quadcopters should cooperate safely to transport load. Formation control of decoupled multi-agent systems has been discussed in [15]. GRASP laboratory at University of Pennsylvania has demonstrated many technological innovations in the domain of autonomous micro UAVs flying inside the constrained environment [16] and performing fantastic feats like cooperatively grasping and transportation [17]. Institute of Control Systems (ICS) at Technical university of Hamburg-Harburg (TUHH) exploited Ho control for formation flight simulation of multiple quadcopters. Institute of Aerospace Information Technology, Würzburg University is working in the domain of quadcopters autonomy for indoor applications mainly to assist the fire fighters against the burning structures. It is fully autonomous in the sense that it does not rely on external signals like GPS and optical tracking system etc. A group of MUAVs is also drawing a lot of attention for 3D aerial maps generation [18] as well as weather monitoring and communication relay [19]. Today we can watch the stunning feats of cooperative micro UAVs flying very accurately and even capable to play ping-pong [20]. More innovative applications are foreseen with advancement in sensor technology, autonomous control techniques, on-board processors and software.

\section{RELATED SENSORS FOR MUAV COOPERATIVE FLIGHT}

We discuss some of the sensors vis-a-vis their purpose. Special emphasis is given to Global Navigation Satellite System (GNSS) receiver and PMD camera.

\subsection{Obstacle Detection and Collision Avoidance}

For MUAVs operating in close vicinity, it is vital for safe operation to avoid mutual collision. One option to tackle this situation may be to install multiple active sensors, like laser scanner or 
ultrasonic sensors [21], covering full $360^{\circ}$ view. An alternate may be to equip the MUAVs with one GPS receiver each exploiting Differential GPS (DGPS) techniques. However for obstacles (like a wall), GPS is not helpful and we are bound to employ multiple sensors on all sides of a quadcopter. Ultrasonic and infra-red sensors or conventional cameras may be the good candidate for this purpose because of their low cost and power consumption. Small radar may also be a good sensor for obstacle detection [22]. Modern technologies like PMD camera and computer vision require on-board high computational power, but can provide better accuracy, resolution and coverage than ultrasonic or infra-red sensors.

\subsection{Attitude and Heading Determination}

Inertial Measurement Unit (IMU) is a traditional sensor to measure the attitude and heading, that is the orientation. However alternate methods also exist exploiting the devices which are not meant for measuring the attitude like converting motor power consumption to Euler angles [23]. Infra-Red (IR) sensors are investigated to be used not only for height above the ground [24] but also for absolute attitude determination [25]. One idea of infra-red attitude sensor is to measure the heat difference between two sensors on one axis to determine the angles of the UAV because the earth emits more IR than the sky. However accuracy directly depends on the baseline length. Attitude can also be determined using multiple GPS antennas [26] or Signal-to-Noise Ratio (SNR) measurements [27]. Though accuracy is dependent on the distance between the sensors. Computer Vision based approaches are also possible. However IMUs are still the most relevant orientation sensor for systems which do not rely on external reference systems like optical tracking. A common approach is to use multiple sensors and then apply data fusion and some

filtering technique like Kalman filter or Particle filter to find true value. Although knowledge of correct attitude and heading is the basis for accurate navigation, but this information is even more critical for cooperating units operating in close locality.

\subsection{GNSS Receiver}

GNSS observables, the pseudo-ranges and carrier phase, are used to determine the position of the receiver. GPS carrier phase can provide quite accurate relative navigation [28]. It can provide accuracy to $\mathrm{cm}$ level, but it is demanding to solve integer ambiguity in real time [29]. Information provided by GPS receiver can be directly used to measure the ground velocity. GPS measurements can be used in real-time as well as in off-line mode e.g. geo-referencing for aerial photography. For post-mission analysis, GPS observables are stored in the form of Receiver INdependent EXchange (RINEX) files [30] and then processed later. Geo-referencing can be verified to some degree using the Google Earth [31].

Differential Global Navigation Satellite System (DGNSS) has been widely used in many applications requiring high accuracy [32]. It is a technique to improve the accuracy of a rover coordinates applying some correction methodology to remove GPS errors. DGPS services are either to be hired through some agency providing correction signals or an indigenous reference station is required to be set-up, where correction factors are computed and then sent to MUAV. Prior knowledge of reference station exact coordinates is mandatory that restricts the use of indigenous reference station in the field. However exact position can be determined using some emerging technologies like Precise Point Positioning (PPP) that does away the need of a base station and is able to provide position solutions at centimeter to decimeter level. It needs to be considered that it requires long initialization time, that is a drawback for real-time applications [33]. Though PPP can serve the purpose to find the exact coordinates of reference station in the field. 
A quick and easy solution to this problem may be the use of Satellite Based Augmentation System (SBAS) services being provided in different regions of the world. It is the cheapest and quickest method (even in challenging environments) to improve the accuracy as these signals are transmitted on L1 frequency and no decoder is required, that is otherwise required in case of RTCM-104 format DGPS. Feature of L1 frequency make the signals usable for low-cost GPS receivers. Performance of European Geostationary Navigation Overlay Service (EGNOS), the European SBAS, in terms of accuracy is $3 \mathrm{~m}$ lateral and $4 \mathrm{~m}$ vertical for open service, while its availability is 99\% [34]. There are many low-cost GNSS receiver providers like u-blox that provides quite precise GNSS receivers e.g. LEA-M8S and NEO-7P (for PPP) being the latest products [35]. DGPS coupled with IMU (for integrated navigation) has been extensively used uniting the merits of both systems [36].

For relative navigation, one is more interested in relative position than absolute position. So in spite of GPS error sources, it can be a good candidate for relative positioning applications [37].

\subsection{PMD Camera}

PMD camera is another emerging technology that is drawing attention during the last few years. It is being used for the domains that have strict time compliance requirements like deployment of an air-bag of a car in case of an accident [38]. PMD camera has the potential to be an excellent sensor for cooperative flight. The authors are not aware of any MUAV exploiting PMD for obstacle detection or relative position and attitude determination. The PMD Nano from pmdtechnologies (Siegen, Germany) is an optimal PMD camera (Figure 4) with regards to price, size and weight that can be used for MUAV. It is capable to measure 3D distances with a resolution of $1 \mathrm{~mm}$ within a range of about $3 \mathrm{~m}$. Use of this emerging technology for MUAVs can introduce new horizons.
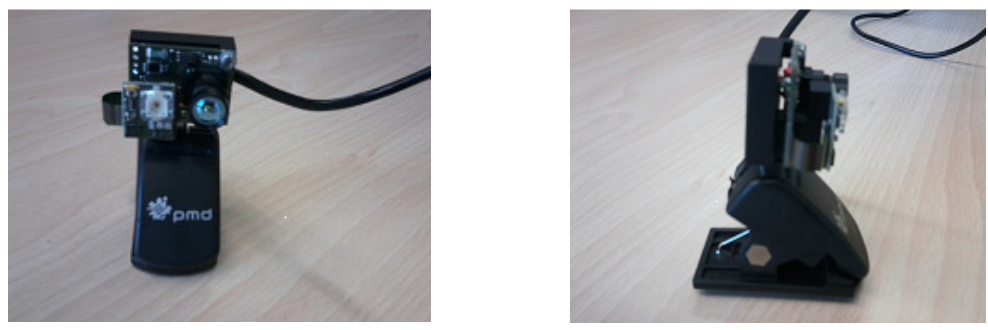

Figure 4. Front view (left) and side view (right) of a PMD camera

Selection of suitable sensors for MUAV cooperative flight forms the basis for the algorithms to be developed. Accuracy of cooperative flight is directly dependent on the sensors installed on the agents.

\section{RELATEd CONTROL TEChNiQUeS AND RELEVANT MATERIAL}

\subsection{Control Techniques}

In order to control the quadcopter, a number of control techniques have been used ranging from the basic controller of PID and LQR [39] to much sophisticated techniques like Non-linear Model Predictive Control (NMPC), Decentralized Model Predictive Control (DMPC) [40], BackStepping and Fuzzy Logic controls etc. The PID controllers have limitations in optimality and robustness. Besides, it is also difficult to tune the parameters under some circumstances [25]. Choice of appropriate control technique depends on the application. Low-cost common-off-the- 
International Journal of Artificial Intelligence \& Applications (IJAIA), Vol. 5, No. 4, July 2014

shelf GPS receivers in combination with the MEMS-based IMUs have been sufficient to maintain the formation of the units meant for mobile communication relay [19] as distances are quite large. However, in case of units operating in close vicinity, control requirements are quite strict. NMPC has been widely used for formation flight of multiple units [41, 42]. MPC can cater for the changes in reference values during the operation and can handle state and control constraints [43]. A solution using NMPC was proposed in [44] where formation flight of multiple UAVs is sustainable even in case of communication failure.

$\mathrm{H} \infty$ control technique, being comparatively simple from implementation point of view, is being exploited at TUHH Germany for cooperative multi agent systems. In [45], a formation controller has been designed to minimize $\mathrm{H} \infty$ performance measure while guaranteeing robust stability. The proposed method is also efficient in the sense that the synthesis procedure is based only on a single agent model, instead of a model of the whole formation with a possibly large number of agents. Graph theory has been used by Murray and Olfati-Saber [46] and Phillip R. Chandler [47] to control cooperating units. A control design based on decentralized Receding Horizon Control (RHC) was proposed [41] for collision-free UAV formation flight. There is generally a trade-off between the intricacy of control technique employed and the level of accuracy achieved there off.

\subsection{MUAV Model Identification}

System identification of a MUAV is considered to be the first step when high accuracy is aimed and sophisticated control techniques are planned to be applied. As MUAV is a highly non-linear system, it is demanding to get an accurate mathematical model to describe its dynamics. Situation becomes even more cumbersome when its configuration also keeps on changing because of different sensors installed. $\mathrm{H} \infty$ loop shaping techniques can be used on small fixed wing UAVs for improvements in noisy or even payload changing circumstances [48].

Not all control techniques require the system to be modelled. Adaptive neural network controller does not require an accurate mathematical model and is suitable for multi-variable flight control. The PID and Neural Network autopilots are non-model based, however the optimality and robustness of the controller cannot be guaranteed. As it is hard to get an accurate non-linear model, a linear model can also be used to approximate the MUAV dynamics [25].

From safety point of view, most of the proposed algorithms require to be simulated before actual flights may be undertaken. It also helps to authenticate the efficacy of the algorithm. However for simulation purpose, a mathematical model of the system under consideration is mandatory. Health of simulation results directly corresponds to accuracy of the modelling. From MUAV point of view, its system can also be identified using MATLAB System Identification Toolbox [49]. The obligation for model identification is application dependent.

\subsection{Distributed Systems}

A modern trend in transportation field is to distribute the electronic systems. Networking has thus become an essential part of system design for connecting independent control units. Communication over the network is vastly growing in modern electronic systems with the advent of smart sensors and actuators. This trend will increase the number of distributed systems with distributed hardware, control and data. It would lead to distribution of functions, control algorithms and data to improve hardware usage. This distribution however poses new problems which are not encountered in monolithic systems e.g. data inconsistency due to transmission delays and communication data packets loss cause inconsistencies in identical functions. Safety related systems are to be taken care of for such scenarios. Design of distributed systems should be able to avoid these so called negative effects of distribution [50]. 
International Journal of Artificial Intelligence \& Applications (IJAIA), Vol. 5, No. 4, July 2014

\subsection{Necessary Information to Share}

A basic question to deal with cooperative flight is that what is the minimum information required to be shared among the units to effect cooperation and to coordinate actions? A flying object has 12 states in general; the position coordinates $(\mathrm{x}, \mathrm{y}, \mathrm{z})$; velocity components $(\mathrm{u}, \mathrm{v}, \mathrm{w})$ along the threeaxes; roll, pitch and yaw angles $(\Phi, \theta, \Psi)$; and the angular rates (p,q,r) measured along the threeaxes [51]. Relative position, velocity and attitude are considered as the minimum variables to be determined for cooperative control. Redundant information sharing is to be avoided in order to reduce the burden on communication.

\subsection{Communication Requirements}

Formation flight control techniques require transmission of states of the leader to the follower that is dependent on some communication data link. The transmissions can be divided into three categories: vehicle telemetry data, commands for the vehicle, and coordination information between vehicles [2]. For applications like live video streaming to the ground station and carrier phase double-difference GPS data in real time, we need to have wide bandwidth communication requirements. For MUAVs, mostly Wi-Fi, blue-tooth [52] and XBee links have been used, each having their own merits and demerits in terms of range, data rate and power requirements. Communication packet loss may occur thereby producing errors in cooperative control of MUAVs and compromising the communication integrity.

An Orthogonal Frequency Division Multiplexing (OFDM) technique with adaptive resource allocation was used at Bleking Institute of Technology, Sweden for small UAV communication with the ground station and between UAVs [53]. [54] discusses the robust stability of multiple cooperative units under time-varying communication topologies and communication delays.

\subsection{Hardware}

Some of the open source quadcopter projects are ArduCopter, AeroQuad and MikroKopter etc. which may be customized as per the needs. Institute of Aerospace Information Technology, Würzburg has constructed its own quadcopter frame which is also being used for educational purpose to verify the control algorithms [55]. Institute of Aircraft Design Stuttgart, for example, is also designing and manufacturing its own UAVs. For computational purposes, mainly on-board computers, FPGAs, Gumstix and micro controllers have been used. Any on-board processor is required to process the data at a faster rate especially for those units operating in close vicinity.

\subsection{Software Tools}

MATLAB/ Simulink environment has been mostly used for simulation of MUAV models, and later hooked up with $\mathbf{C} / \mathbf{C}++$ programming language (used predominantly for real time systems). MATLAB Real Time Workshop (RTW) can be used for automatic generation of $\mathbf{C}$ code directly from Simulink models. Similarly MATLAB Coder toolbox can also be used for generation of standalone $\mathbf{C}$ and $\mathbf{C}++$ code from MATLAB code. Some research groups are using Real time Operating System (RTOS) for quadcopters e.g. $\mu \mathrm{C} / \mathrm{OS}-\mathrm{II}^{\mathrm{TM}}$ [56], Tiptoe [57] and FreeRTOS [58]. Real-time On-board Dependable Operating System (RODOS) [59], developed by Deutsches Zentrum für Luft- und Raumfahrt (DLR) Germany and Institute of Information Technology Würzburg University, has been successfully applied on operational satellites and quadcopters. It is written in $\mathrm{C}++$ and emphasis has been placed on simplicity. It has the potential to be utilized for the constellation of MUAVs. Selection of appropriate software depends on the application requirement. 
International Journal of Artificial Intelligence \& Applications (IJAIA), Vol. 5, No. 4, July 2014

RTKLIB [60] software is one of the most popular in the domain of open-source GNSS precise positioning and has been used extensively for low-cost GNSS receivers [61]. It is a compact and portable program library written in $\mathbf{C}$ to provide a standard platform for Real Time Kinematics (RTK) applications. It is capable not only for single positioning but also for DGPS, PPP and relative navigation etc. The software has good capabilities for real-time as well as post-mission analysis. It is a precise positioning technology, with which users can obtain cm-level accuracy of the position in real-time by processing carrier-phase measurements of GPS signals [62].

Accuracy of cooperative behaviour is based on the control technique selected and the sensors used. Implementation of effective distributed control technique is also dependent on integrity of communication data.

\section{STATE OF THE ART}

Today small quadcopters are able to perform basic construction while assembling the parts. MUAVS can perform aggressive manoeuvres requiring very high precision. Quadcopters can even play the musical instruments in a coordinated fashion. They can grasp and transport the load using multiple units operating in harmony [17]. An indoor formation flying of 20 micro quadcopters performing coordinated actions has already been materialized. ETH Zurich exhibits state of the art coordination of MUAVs like cooperative construction, inverted pendulum balancing and ball juggling with a net utilizing multiple MUAVs. The Institute has also designed a 'Distributed Flight Array', a flying platform consisting of multiple autonomous single propeller vehicles that are able to drive, dock with their peers, and fly in a coordinated fashion. But all those systems rely on a high accurate external positioning system like optical tracking.

Traditional quadcopters have fixed-pitch propellers that can generate the thrust only in one direction. Variable pitch quadcopters have been designed capable of accelerating and decelerating at a very fast rate and flight demonstrated for inverted flight and aggressive manoeuvres [63]. Such platforms have higher control bandwidth. Quadcopters have tremendous potential for their growth keeping in view their future applications. Miniaturization of these vehicles will still open new vistas for many other applications.

\subsection{Areas Requiring Attention}

In case of MUAVs, we are to restrict ourselves to the sensors and hardware which have small volume, weight and power consumption. Real time on-board voluminous data processing is mandatory as may be required in case of PMD camera that necessitates the need of more powerful on-board processors. There is still a lot of room for improvement in the domain of more sophisticated algorithms for distributed task allocation and autonomous decision making by the agents keeping in view the computational intensity. Limited bandwidth of communication data link is another limitation that poses new challenges for real-time implementation of some techniques like GPS carrier-phase double-difference and live video streaming from MUAV. Robust algorithms are required to cater for communication packets loss.

Autonomy is still a challenging field of research, when it comes to substitute external positioning systems with on-board sensors, which suffer from low accuracy because of limited resources in size, weight, power, battery capacity and processing. But these problems are unavoidable when a MUAV needs to fly autonomously in unknown, GPS-denied and adverse (e.g. smoke) environments. This is an up to date field of research, as MUAVs are being designed to help fire fighters inside burning buildings. Docking for air refuelling for autonomous UAVs is still an open topic for future research. MUAVs potential for agriculture applications may be quite interesting 
International Journal of Artificial Intelligence \& Applications (IJAIA), Vol. 5, No. 4, July 2014

for agricultural sectors e.g. spraying the insecticides to prevent the humans from toxicity.

Endurance of MUAV is limited by the life of battery so these can operate within a short distance from the launching point. Some alternatives to extend the mission duration have been suggested like automated battery changing/ charging capability for autonomous UAV beyond the life of a single UAV battery [64]. Batteries with extended life will open the door for many other useful applications. Exploitation of solar energy [65] to enhance the MUAV endurance may also be investigated.

As it is quite laborious to model all the dynamics of VTOLS flying in close formation, adaptive and robust control techniques may be explored to their full potential to cater for such scenarios. These control techniques may also be quite valuable to control swarm behaviour. Collision avoidance for a swarm of MUAVs is still an area that requires attention. In this context, velocity matching and flock joining algorithms may be improved. Fault tolerant control methods may further increase the integrity and reliability of MUAVs. Convertible MUAVs can be another interesting area for researchers thereby combining the merits of fixed wings as well as VTOLs. New air vehicle structures may be proposed and developed for this purpose. Flapping wing MUAVs while exploiting synthetic muscles is another domain of research to mimic the birds and to perform novel manoeuvres. Multi-discipline research may also be undertaken to develop biological-inspired systems while learning from nature e.g. optic flow vision of flying insects. Low Reynolds number aerodynamics for small fixed wing airplanes also needs to be focussed.

\section{CONCLUSIONS}

MUAVs have already replaced manned aircraft in many fields and are even capable to perform novel assignments which cannot be performed by manned platforms. Utilization of MUAVs is expected to rise steadily particularly for remote sensing missions while exploiting emerging technologies. Their utilization is foreseen ranging from emergency situations handling e.g. fire fighting, volcanoes monitoring etc. to routine tasks like postal and parcel services, film shooting, patrolling and wild life survey etc. Incorporation of a robotic arm to a MUAV will enhance their applications to further extent. Unmanned is therefore unmatched. There is still a lot to explore for this fascinating field. It definitely has the potential to serve the humanity in an even better and advanced fashion.

\section{ACKNOWLEDGEMENTS}

This publication was funded by the German Research Foundation (DFG) and the University of Wuerzburg in the funding programme Open Access Publishing.

Scholarship to Qasim Ali for this research was financed by Higher Education Commission (HEC) of Pakistan and administered by Deutscher Akademischer Austausch Dienst (DAAD) Germany. The author would like to thank Dr. Zeeshan Ahmed (University of Wuerzburg, Germany) for initial review and stylistic corrections to this paper.

\section{REFERENCES}

[1] Shima, Tal, and Steven J. Rasmussen, eds. "UAV cooperative decision and control: challenges and practical approaches." Vol. 18. SIAM, 2009.

[2] Beard, Randal W., et al. "Decentralized cooperative aerial surveillance using fixed-wing miniature UAVs." Proceedings of the IEEE 94.7 (2006): 1306-1324.

[3] Kumar, Vijay, and Nathan Michael. "Opportunities and challenges with autonomous micro aerial vehicles." The International Journal of Robotics Research 31.11 (2012): 1279-1291.

[4] Flores, Gerardo, and Rogelio Lozano. "Transition flight control of the quad-tilting rotor convertible 
International Journal of Artificial Intelligence \& Applications (IJAIA), Vol. 5, No. 4, July 2014

MAV." Unmanned Aircraft Systems (ICUAS), 2013 International Conference IEEE.

[5] Chao, Zhou, et al. "UAV formation flight based on nonlinear model predictive control." Mathematical Problems in Engineering (2012).

[6] Ren, Wei, Randal W. Beard, and Timothy W. McLain. "Coordination variables and consensus building in multiple vehicle systems." Cooperative Control. Springer Berlin Heidelberg, 2005. 171-188.

[7] Seo, Joongbo, Chaeik Ahn, and Youdan Kim. "Controller design for UAV formation flight using consensus based decentralized approach." Proceedings of AIAA Infotech@ Aerospace Conference, AIAA Press. 2009.

[8] Wagner, Geno, et al. "Flight test results of close formation flight for fuel savings." AIAA Atmospheric Flight Mechanics Conference and Exhibit. 2002.

[9] Ardaens, J. S., and S. D’Amico. "Formation flying testbed." DLR-GSOC TN (2009): 09-01.

[10] How, Jonathan, Ellis King, and Yoshiaki Kuwata. "Flight demonstrations of cooperative control for UAV teams." AIAA 3rd unmanned unlimited technical conference, workshop and exhibit. 2004.

[11] Park, Chan-Woo. "Precise relative navigation using augmented CDGPS." Diss. Stanford University, 2001.

[12] Park, Sanghyuk. "Avionics and control system development for mid-air rendezvous of two unmanned aerial vehicles." Diss. Massachusetts Institute of Technology, 2004.

[13] Hexmoor, Henry, Shahram Rahimi, and Jody T. Little. "Coordinated UAV manoeuvring flight formation." Informatica (03505596) 33.3 (2009).

[14] Ross, Steven M. "Formation flight control for aerial refueling." No. AFIT/GAE/ENY/06-M35. Air Force Inst of Tech Wright-Patterson AFB OH School of engineering and Management, 2006.

[15] Sreenath, Koushil, Taeyoung Lee, and Vijay Kumar. "Geometric control and differential flatness of a quadrotor UAV with a cable-suspended load." IEEE Conference on Decision and Control (CDC) 2013.

[16] Michael, Nathan, et al. "The GRASP multiple micro-UAV testbed." Robotics \& Automation Magazine, IEEE 17.3 (2010): 56-65.

[17] Mellinger, Daniel, et al. "Cooperative grasping and transport using multiple quadrotors." Distributed autonomous robotic systems. Springer Berlin Heidelberg, 2013. 545-558.

[18] Parunak, H. Van Dyke, Sven Brueckner, and James J. Odell. "Swarming coordination of multiple UAV's for collaborative sensing." Proceedings of Second AIAA "Unmanned Unlimited" Systems, Technologies, and Operations Conference. 2003.

[19] Angermann, Michael, Martin Frassl, and Michael Lichtenstern. "Autonomous formation flying of micro aerial vehicles for communication relay chains." San Diego, CA (2011).

[20] Muller, Mark, Sergei Lupashin, and Raffaello D'Andrea. "Quadrocopter ball juggling." Intelligent Robots and Systems (IROS), 2011 IEEE/RSJ International Conference on. IEEE, 2011.

[21] Gageik, Nils, Thilo Müller, and Sergio Montenegro. "Obstacle detection and collision avoidance using ultrasonic distance sensors for an autonomous quadrocopter." University Of Würzburg, Aerospace Information Technology (Germany), Würzburg September (2012).

[22] Moses, Allistair, Matthew J. Rutherford, and Kimon P. Valavanis. "Radar-based detection and identification for miniature air vehicles." Control Applications (CCA), 2011 IEEE International Conference on. IEEE, 2011.

[23] Moyano Cano, Javier. "Quadrotor UAV for wind profile characterization." (2013).

[24] Gageik, Nils, Julian Rothe, and Sergio Montenegro. "Data fusion principles for height control and autonomous landing of a quadrocopter." (2012).

[25] Chao, HaiYang, YongCan Cao, and YangQuan Chen. "Autopilots for small unmanned aerial vehicles: a survey." International Journal of Control, Automation and Systems 8.1 (2010): 36-44.

[26] Vaillon, L., et al. "Attitude determination using GPS: Multipath reduction through GPS antenna design." Spacecraft Guidance, Navigation and Control Systems. Vol. 425. 2000.

[27] Lightsey, E. Glenn, and Jared Madsen. "Three-axis attitude determination using global positioning system signal strength measurements." Journal of guidance, control, and dynamics 26.2 (2003): 304310 .

[28] Li, Rui, et al. "Simulation platform for relative navigation using GPS carrier phase measurements for satellite formation flying missions." IGNSS Symposium 2011.

[29] Olsen, Eric A., Chan-Woo Park, and Jonathan P. How. "3D formation flight using differential carrierphase GPS sensors." Navigation 46.1 (1999): 35-48.

[30] Zhang, Wen, Mounir Ghogho, and L. Enrique Aguado. "GPS short-distance baseline estimation from RINEX files under MATLAB environment." Proc. of the 13th IAIN World Congress and Exhibition. 2009. 
International Journal of Artificial Intelligence \& Applications (IJAIA), Vol. 5, No. 4, July 2014

[31] Potere, David. "Horizontal positional accuracy of Google Earth's high-resolution imagery archive." Sensors 8.12 (2008): 7973-7981.

[32] Olsen, Eric Alan, and Jonathan P. Adviser-How. "GPS sensing for formation flying vehicles." Stanford University, 2000.

[33] European Space Agency (ESA). Precise Point Positioning (PPP), February 2012, http://www.navipedia.net/index.php/PPP.

[34] European Space Agency (ESA). EGNOS, February 2012, http://www.navipedia.net/index.php/EGNOS Performances.

[35] u-blox, http://www.u-blox.com/en/gps-modules/pvt-modules.html

[36] Wang, Jinling, et al. "Integration of GPS/INS/Vision sensors to navigate unmanned aerial vehicles." International Society for Photogrammetry and Remote Sensing (ISPRS) Congress. 2008.

[37] Tobe'Corazzini, Andrew Robertson, et al. "GPS sensing for spacecraft formation flying." (1997).

[38] Schwarte, R., et al. "New powerful sensory tool in automotive safety systems based on PMDtechnology." Advanced Microsystems for Automotive Applications 2000. Springer Berlin Heidelberg, 2000. 181-203.

[39] Argentim, Lucas M., et al. "PID, LQR and LQR-PID on a quadcopter platform." Informatics, Electronics \& Vision (ICIEV), 2013 International Conference on IEEE, 2013.

[40] Richards, Arthur, and Jonathan How. "Decentralized model predictive control of cooperating UAVs." Decision and Control, 2004. CDC. 43rd IEEE Conference on. Vol. 4. IEEE, 2004.

[41] Borrelli, Francesco, Tamás Keviczky, and Gary J. Balas. "Collision-free UAV formation flight using decentralized optimization and invariant sets." Decision and Control, 2004. CDC. 43rd IEEE Conference on. Vol. 1. IEEE, 2004.

[42] Chao, Zhou, et al. "UAV formation flight based on nonlinear model predictive control." Mathematical Problems in Engineering (2012).

[43] Wang, Xiaohua, Vivek Yadav, and S. N. Balakrishnan. "Cooperative UAV formation flying with obstacle/collision avoidance." Control Systems Technology, IEEE Transactions on 15.4 (2007): 672679.

[44] Shin, Hyo-Sang, Min-Jea Thak, and Hyoun-Jin Kim. "Nonlinear model predictive control for multiple UAVs formation using passive sensing." International Journal of Aeronautical and Space Sciences 12.1 (2011): 16-23.

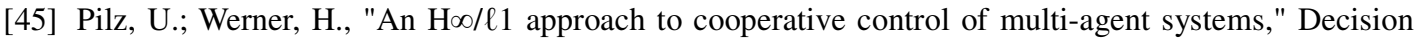
and Control (CDC), 2012, pp.5930 - 5935, 10-13 Dec. 2012 DOI: 10.1109/CDC.2012.6426879

[46] Olfati-Saber, Reza, and Richard M. Murray. "Consensus problems in networks of agents with switching topology and time-delays." Automatic Control, IEEE Transactions on 49.9 (2004): 15201533.

[47] Chandler, Phillip R., et al. "Complexity in UAV cooperative control." American Control Conference, 2002. Proceedings of the 2002. Vol. 3. IEEE, 2002.

[48] Sadraey, M., and R. Colgren. "Two DOF robust nonlinear autopilot design for a small UAV using a combination of dynamic inversion and $\mathrm{H} \infty$ loop shaping." AIAA Guidance Navigation and Control Conference. Vol. 2. 2005.

[49] Lehnert, Christopher, and Peter Corke. " $\mu$ AV-Design and implementation of an open source micro quadrotor." Proceedings of Australasian Conference on Robotics and Automation, 2-4 Dec 2013, University of New South Wales, Sydney Australia

[50] Kiencke, Uwe, et al. "The impact of automatic control on recent developments in transportation and vehicle systems." Annual reviews in control 30.1 (2006): 81-89.

[51] Stevens, Brian L., and Frank L. Lewis. "Aircraft control and simulation." Vol. 2. New York: Wiley, 2003.

[52] Gageik, Nils, Michael Strohmeier, and Sergio Montenegro. "Waypoint flight parameter comparison of an autonomous UAV." International Journal of Artificial Intelligence \& Applications 4.3 (2013).

[53] Mahmood, Shahid. "Unmanned Aerial Vehicle (UAV) communications." (2007).

[54] Popov, Andrey, and Herbert Werner. "Robust stability of a multi-agent system under arbitrary and time-varying communication topologies and communication delays." IEEE transactions on automatic control 57.9 (2012): 2343-2347.

[55] Gageik, Nils, Atheel Redah, and Sergio Montenegro. "Avionics control systems for education and development." INTED 2012 Proceedings : 935-942.

[56] Sababha, Belal H., Hong Chul Yang, and Osamah A. Rawashdeh. "An RTOS-based run-time reconfigurable avionics system for UAVs." AIAA Infotech@ Aerospace 2010.

[57] Craciunas, S., et al. "The JAviator: A high-payload quadrotor UAV with high-level programming 
International Journal of Artificial Intelligence \& Applications (IJAIA), Vol. 5, No. 4, July 2014

capabilities." Proc. GNC (2008).

[58] Tosteberg, Joakim, and Thomas Axelsson. "Development of a wireless video transfer system for remote control of a lightweight UAV." (2012).

[59] Montenegro, Sergio, and Lutz Dittrich. "Network centric core avionics." 7th Symposium on Small Satellites for Earth Observation. 2009.

[60] Takasu, T. "RTKLIB: An open source program package for GNSS positioning." http://www.rtklib.com/prog/rtklib_2. 4.1. zip (2011).

[61] Wiśniewski, B., K. Bruniecki, and M. Moszyński. "Evaluation of RTKLIB's positioning accuracy using low-cost GNSS receiver and ASG-EUPOS." TransNav: International Journal on Marine Navigation and Safety of Sea Transportation 7.1 (2013).

[62] Takasu, Tomoji, and Akio Yasuda. "Development of the low-cost RTK-GPS receiver with an open source program package RTKLIB." International Symposium on GPS/GNSS, International Convention Center Jeju, Korea. 2009.

[63] Cutler, Mark, and Jonathan P. How. "Actuator constrained trajectory generation and control for variable-pitch quadrotors." AIAA Guidance, Navigation, and Control Conference (GNC). 2012.

[64] Toksoz, Tuna. "Design and implementation of an automated battery management platform." Diss. Massachusetts Institute of Technology, 2012.

[65] Della Penna, Giuseppe, et al. "Resource-optimal planning for an autonomous planetary vehicle." International Journal of Artificial Intelligence \& Applications 1.3 (2010).

\section{Authors}

M.S. Qasim Ali is a $\mathrm{PhD}$ student at the Chair Aerospace Information Technology at the University of Wuerzburg, Germany. He received his Master degree in Control Systems from Air University Islamabad in 2010.

Dipl.-Ing. Nils Gageik is working as a research assistant and $\mathrm{PhD}$ student at the Chair Aerospace Information Technology at the University of Wuerzburg, Germany. He received his Diplom in Computer Engineering from RWTH Aachen University in 2010

Prof. Dr.-Ing. Sergio Montenegro is holder of the Chair Aerospace Information Technology at the University of Wuerzburg, Germany
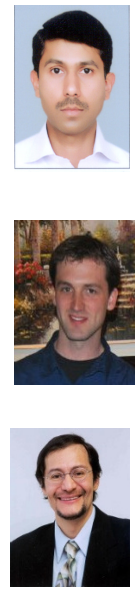